2017-02-22

\title{
Rapid detection of snakes modulates spatial orienting in infancy
}

\author{
Bertels, J
}

http://hdl.handle.net/10026.1/9953

10.1177/0165025417693955

International Journal of Behavioral Development

SAGE Publications

All content in PEARL is protected by copyright law. Author manuscripts are made available in accordance with publisher policies. Please cite only the published version using the details provided on the item record or document. In the absence of an open licence (e.g. Creative Commons), permissions for further reuse of content should be sought from the publisher or author. 


\title{
Rapid detection of snakes modulates spatial orienting in infancy
}

\author{
Julie Bertels, ' Clémence Bayard, ${ }^{2}$ Caroline Floccia, ${ }^{3}$ \\ and Arnaud Destrebecqz'
}

\begin{abstract}
Recent evidence for an evolved fear module in the brain comes from studies showing that adults, children and infants detect evolutionarily threatening stimuli such as snakes faster than non-threatening ones. A decisive argument for a threat detection system efficient early in life would come from data showing, in young infants, a functional threat-detection mechanism in terms of "what" and "where" visual pathways. The present study used a variant of Posner's cuing paradigm, adapted to 7-I I-month-olds. On each trial, a threat-irrelevant or a threatrelevant cue was presented (a flower or a snake, i.e., "what"). We measured how fast infants detected these cues and the extent to which they further influenced the spatial allocation of attention ("where"). In line with previous findings, we observed that infants oriented faster towards snake than flower cues. Importantly, a facilitation effect was found at the cued location for flowers but not for snakes, suggesting that these latter cues elicit a broadening of attention and arguing in favour of sophisticated "what-where" connections. These results strongly support the claim that humans have an early propensity to detect evolutionarily threat-relevant stimuli.
\end{abstract}

\section{Keywords}

attention, cuing paradigm, infancy, snake, threat

Snakes and primates co-exist for millions of years, with snakes being the first of the major predators of primates (Isbell, 2006, 2009). Effective processes of snake detection may have thus been fostered through natural selection in primates as such ability would allow a better defensive behaviour. A fear module could have evolved in mammals' brain to assist them in responding adequately to recurrent survival threat (Öhman \& Mineka, 2003), in particular to threats represented by reptiles, of which snakes are a prototypical exemplar. Going one step further, it has been suggested that this evolutionary ancient predator-prey relationship played a significant role in the evolution of the primates' visual system. The Snake Detection Theory posits that the vital need to rapidly detect snakes shaped primates' brain such that they developed acute perceptual abilities (Isbell, 2006, 2009).

Recent studies have consistently demonstrated that humans are remarkable snake detectors. Using a visual search task, Öhman, Flykt, and Esteves (2001) were the first to report that adults are faster to detect a picture of a snake in an array of flower pictures than vice versa, supporting the claim that snakes capture attentional resources. This finding has been replicated many times (e.g., Lipp, Derakshan, Waters, \& Logies, 2004; LoBue \& Mathews, 2014; LoBue, Mathews, Harvey, \& Stark, 2014; Soares, Lindström, Esteves, \& Öhman, 2014), and extended to young children who in all likelihood have at the very most little experience and knowledge about the dangerousness of snakes (Hayakawa, Kawai, \& Masataka, 2011; LoBue \& DeLoache, 2008, 2011; Masataka, Hayakawa, \& Kawai, 2010; Penkunas \& Coss, 2013a, 2013b). Although preschool children are admittedly less experienced with snakes than adults, a stronger and more decisive argument for an inborn threat (and snakes in particular) detection system would come from infants and lab-reared monkeys. A few recent studies point in that direction.
Lab-reared macaque monkeys with no prior experience with snakes have the same propensity as humans to faster detect a snake among flowers than a flower among snakes (Shibasaki \& Kawai, 2009). Crucially, Van Le et al. (2013) recently reported that pulvinar neurons in the macaque brain respond selectively to images of snakes, supporting the existence of a neurobiological substrate for the rapid detection of snakes in primates. In order to investigate the predisposition to rapidly detect snakes in human infants, in two recent studies, 7-18-month-olds were presented with pairs of pictures, one threat-relevant (e.g., a snake) and the other threat-irrelevant (e.g., a flower), shown side by side (DeLoache \& LoBue, 2009; LoBue \& DeLoache, 2010). Supporting the claim that human infants detect snakes rapidly, infants turned faster toward the snake than the threatirrelevant picture of the pair. These results provide a first support for the existence of an attentional bias toward snakes in infancy. Together with the findings in lab-reared macaque monkeys, they strongly suggest that enhanced visual detection of snakes would not depend on prior experience with these

\footnotetext{
' ULBabyLab, Consciousness, Cognition and Computation Group (CO3), Center for Research in Cognition and Neurosciences (CRCN), Université libre de Bruxelles, Brussels, Belgium

${ }^{2}$ Centre Nationnal de la Recherche Scientifique, Département Parole et Cognition, Gipsa-Lab, Grenoble Université, Grenoble, France

${ }^{3}$ University of Plymouth, School of Psychology, Plymouth, UK
}

\section{Corresponding author:}

Julie Bertels, Center for Research in Cognition and Neurosciences (CRCN), Université libre de Bruxelles, Avenue F.D. Roosevelt, 50 - CP I9I, Brussels, Belgium.

Email: jbertels@ulb.ac.be 
animals, and support the existence of an inborn mechanism for the rapid detection of an evolutionary threat.

The underlying explanation for these detection mechanisms is that primates would have evolved visual templates for recognizing threatening animals such as snakes and spiders (Rakison \& Derringer, 2008). These templates would integrate low-level features and forms of these threatening animals, and attract infants' attention to their real-world counterparts. While the critical lowlevel perceptual feature for detecting snakes would be their curvilinear and coiled shape (LoBue, 2014; LoBue \& DeLoache, 2011), the oval body connected to curved legs characteristic of spiders would be crucial for rapidly detecting these arachnids (Rakison \& Derringer, 2008). Interestingly, it has been recently suggested that humans would also have evolved auditory templates to rapidly detect threats signalled by sound features (Erlich, Lipp, \& Slaughter, 2013). The evolved predisposition to detect threat would thus not be limited to the visual modality.

Importantly, while human infants would detect snakes particularly rapidly, they would not innately fear them. Several studies using behavioural and physiological measures demonstrated that infants do not show actual fear when exposed to movies displaying snakes in motion (DeLoache \& LoBue, 2009; Thrasher \& LoBue, 2016). Nevertheless, humans, and more generally primates, would be prepared to develop responses of fear towards snakes (DeLoache \& LoBue, 2009; Öhman \& Mineka, 2001, 2003). In fact, the detection bias towards snakes in primates would facilitate learning of an association between the presence of a snake and a fearful reaction (Öhman \& Mineka, 2001, 2003; Rakison \& Derringer, 2008).

To be fully functional, threat detection would bear on the two parallel streams of processing composing the visual system: the "what" and the "where" subsystems (Milner \& Goodale, 1995). The "what" subsystem is involved in the recognition and identification of visual objects. Also referred to as the "ventral stream", it extends from the primary visual cortex (V1) to portions of the temporal cortex. The "where" subsystem subtends the processing and attending to the objects' spatial location. This is also called the "dorsal stream", and it projects from V1 to regions of the parietal cortex. Both subsystems develop early in life, although different aspects emerge at different ages (Johnson, Mareschal, \& Csibra, 2008).

Evidence for an early development of the "what" visual pathway comes, for instance, from face processing studies: newborns look longer at face-like than non-face-like stimuli (Johnson, Dziurawiec, Ellis, \& Morton, 1991), and at their mother's face than at another woman's (Bushneil, Sai, \& Mullin, 1989). Infants' faster processing of a snake than of a non-threatening picture (DeLoache \& LoBue, 2009; LoBue \& DeLoache, 2010) also supports the functionality of the "what" visual pathway.

Regarding the "where" pathway, many studies using spatial cuing paradigms have documented infants' ability to shift attention from one location to another from birth onwards (Valenza, Simion, \& Umiltà, 1994). Cuing of visual attention seems effective by 4 months of age, with infants (like adults) showing both facilitation and inhibition of response to a cued spatial location depending on the Stimulus Onset Asynchrony (SOA, Johnson, 1994). Timing parameters and the distribution of attention across the visual field (linked to cue/target eccentricity) are nevertheless highly decisive in eliciting such shifts. Their influence depends on the infant's age (Harman, Posner, Rothbart, \& Thomas-Thrapp, 1994; Johnson \& Tucker, 1996). In fact, the ability to orient attention in the visual space develops in the first year of life, in parallel with the ability to program eye movements (Harman et al., 1994; Johnson, 1994). In particular, the preference for novel, uncued locations develops between 3 and 6 months of age (Harman et al., 1994) and depends on the target eccentricity: while 3-month-olds demonstrate such a preference only for short target eccentricities, 6-month-olds, who are better able to program eye movements, orient their attention preferentially to uncued locations also at higher target eccentricities (Clohessy, Posner, Rothbart, \& Vecera, 1991).

Some degree of integration between both subsystems are already present in early infancy (Johnson et al., 2008). Coherently, the two pathways are richly interconnected in the developing brain (Stiles, Paul, \& Ark, 2008). The existence of an early functional link between them is supported by recent studies demonstrating that social cues affect the orientation of spatial attention. For instance, the direction of an adult's eye gaze can bias 4-month-old infants' attention towards the corresponding location, and cause enhanced processing of any object presented thereon (Reid, Striano, Kaufman, \& Johnson, 2004). Threat-relevant facial expressions have also been found to hamper infants' attentional disengagement processes (Peltola, Leppanen, Palokangas, \& Hietanen, 2008).

In the present study, we wanted to establish further the existence of a threat-dependent functional link between the "what" and "where" subsystems. Specifically, we examined whether the detection of unfamiliar, non-social threat-relevant stimuli like snakes (whose processing bears on the "what" subsystem) at specific locations modulates the subsequent processing of stimuli appearing either at the same or different locations (those processes belong to the "where" subsystem). As argued elsewhere (Bertels, Kolinsky, $\&$ Morais, 2010), an effective interaction between subsystems would improve the ecological adaptation of any organism. Threat detection would modulate the orientation of attention towards the relevant locations in the environment and therefore foster proper reactions to the to-be-attended stimuli. The existence of such a functional link was investigated in infants from 7 months old, since snake detection biases have been documented from this age on (DeLoache \& LoBue, 2009; LoBue \& DeLoache, 2010). Moreover, at this age infants' attentional orienting abilities are well developped (Johnson, 1994).

We used the spatial cuing paradigm in which, originally, a peripheral cue is followed by a target presented either in the same or in another, opposite location (Posner, 1980). This paradigm has been widely used to study attentional orienting. Attention allocation might indeed be inferred based on response latencies to targets presented at cued or uncued locations. We adapted the paradigm in two ways. First, the cue may have a threat-related content, to examine whether cue facilitation effects (i.e., faster responses to targets presented at the same location as the preceding cue than at the opposite location) are modulated by the nature of the cue (Stormark, Nordby, \& Hugdahl, 1995). In the present study, cues were presented peripherally and consisted of pictures of either snakes (in threat-relevant trials) or flowers (in threat-irrelevant trials).

Second, we adapted the task to infants so as to study their attentional shifts through saccades in response to a stimulus (Clohessy et al., 1991). In each trial, we presented a central attention getter (a blinking star) between the cue and the target in order to reorient their attention to the centre of the screen. We also used a bilateral target (two checkerboard patterns, one on the left and one on the right) to be able to record (1) the latency of the first saccade following the targets' presentation and (2) the direction of the first saccade, depending on the location and nature of the preceding cue. Cue facilitation effects could then result in faster saccades and more 
first looks oriented toward the cued than toward the uncued target location.

We had two main predictions derived from the "what-where" connection hypothesis. First, in line with previous studies suggesting that infants preferentially allocate their attention to threatrelevant than to threat-irrelevant stimuli (DeLoache \& LoBue, 2009; LoBue \& DeLoache, 2010), we expected faster orienting towards snake than flower cue locations. First looks should then be faster when directed towards snake than towards flower cues. Second, we predicted that the presentation of a snake vs. a flower would modulate cue facilitation effects. Specifically, we expected facilitation effects when threat-irrelevant flower cues were presented, namely faster and more frequent first looks toward the cued than toward the uncued target. In contrast, the presentation of snake cues might result in two different types of cuing effects. First, facilitation effects observed with flower cues may be cancelled, if not reversed, after a snake cue. Indeed, from an evolutionary point of view, it would not be adaptive for infants' attention, after having been disengaged from the snake location, to keep on shifting back to that location. Rather, new locations should be explored to detect the presence of additional danger and consider escaping. Such an abolition of cuing effects by threatening stimuli has been observed previously in adults (Bertels, Kolinsky, Bernaerts, \& Morais, 2011). Another possibility is that facilitation effects would be enhanced when a snake cue is presented. Looking back at the snake location would indeed be relevant as to monitor the snake. Preferential processing of targets appearing at previous snake locations has been reported before, but only when attention was not driven back to a central location before the onset of the target (Lipp \& Derakshan, 2005).

\section{Method}

\section{Participants}

Nineteen $7-11$-month-old healthy full term infants ${ }^{1}$ ( 5 boys) with no prior experience of snakes made up the final sample (mean age $=284$ days, range $=223-361)$. Three additional infants were excluded from the study due to a looking bias: their first gaze was directed towards the left $(n=2)$ or right target $(n=1)$ on every trial.

\section{Apparatus}

Stimulus presentation and timing were controlled using the Psychophysics Toolbox software (Brainard, 1997). Stimuli were presented through a 55' LCD digital TV screen. Infants' looking behaviour was monitored with a video camera located above the screen. Infants' looking behaviour as well as the specific experimental display presented to each participant were recorded and synchronized for off-line analyses by Media Recorder 2.5 (Noldus, The Netherlands).

\section{Stimuli and procedure}

Each infant was tested individually while seated on the parent's lap $100 \mathrm{~cm}$ from the TV screen, in a separate 3-sided enclosure of a dimly lit room.

Each trial began with the presentation of an attention getter on the centre of the screen, consisting of a blinking star $(20.3 \times$ $22.8 \mathrm{~cm}$ ) accompanied by an attractive sound (Figure 1). Once the

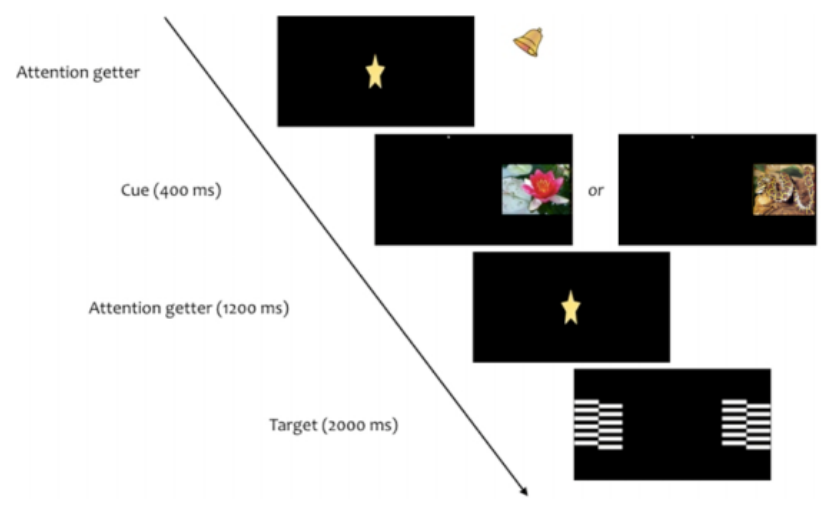

Figure I. Time course of a trial. Each trial begins with the presentation of an attention-getter (a centrally presented blinking star accompanied by an attractive sound). Once the infant is looking at it, a cue is presented for $400 \mathrm{~ms}$, either on the right (as depicted here) or on the left of the screen. The cue is either a picture of a flower (threat-irrelevant trials) or a snake (threat-relevant trials). The central blinking star is presented again for $1200 \mathrm{~ms}$ and followed by the bilateral target, consisting of two checkerboard patterns (one on the left and one on the right of the screen) flickering at a $10-\mathrm{Hz}$ frequency.

infant's attention focused on the star, the experimenter initiated the presentation of the cue. The cue consisted of one amongst eight brightly coloured pictures of snakes and flowers in their natural background used in a previous study (LoBue \& DeLoache, 2008) for $400 \mathrm{~ms}$, peripherally presented on the left or right with the same likelihood, at an eccentricity of $30^{\circ}$ visual angle. Each cue measured $29.9 \times 22.8 \mathrm{~cm}$. The duration and the eccentricity of the cues were chosen as to foster eye movements towards their location (i.e., overt attentional orienting, see e.g., Clohessy et al., 1991). Then, the central blinking star was presented again for $1200 \mathrm{~ms},{ }^{2}$ silently. It aimed at reorienting the infant's attention to the centre of the screen before the 2000-ms presentation of the bilateral target consisting of two checkerboard patterns (one on the left and one on the right, both measuring $29.9 \times 22.8 \mathrm{~cm}$ and presented at $30^{\circ}$ ) flickering by alternating their contrast polarity at a $10-\mathrm{Hz}$ frequency.

Each infant was presented with four 8-trial blocks. In each block, a different cue (either a snake or a flower) was presented on every trial, which resulted in four threat-relevant and four threatirrelevant trials, half on the left and half on the right (counterbalanced across cue types). Trials were randomly presented.

\section{Coding}

Looking times for each infant were coded frame-by-frame (i.e., $25 \mathrm{~ms}$ intervals) by a graduate student trained by the first author, using The Observer XT11 (Noldus, The Netherlands). Coding was blind since it was made separately on videos of the infant's face and on videos of the experimental display he/she was presented with. The videos of the infant's face and of the experimental display were synchronized after the coding. The first author independently coded about $10 \%$ of the data. Inter-coder reliability was $96 \%$.

Three variables of interest, corresponding to three different looking-behaviours occurring in sequence, were measured: (1) the latency to look at the cue, namely the amount of time from the onset of the cue to the infant's first look toward the cue; ${ }^{3}$ (2) the latency to look at the target, namely the amount of time from the onset of the 
Table I. Mean latencies of the first look to the target as a function of the direction of this first look and of the type of cue presented just before.

\begin{tabular}{lcc}
\hline & \multicolumn{2}{c}{ Direction of first look } \\
\cline { 2 - 3 } & Cued target & Uncued target \\
\hline Flower cue & $427[391,464]$ & $437[402,472]$ \\
Snake cue & $414[366,462]$ & $456[410,502]$ \\
\hline
\end{tabular}

Note. $n=19.95 \% \mathrm{Cls}$ are in brackets.

bilateral target to the infant's first look toward one of the target; and (3) the percentage of first gaze oriented toward the "cued" over the "uncued" target, namely the proportion of trials in which infants looked first at the target presented at the cue location.

In each trial, each variable of interest was measured only if the prior variables of interest could also be measured within the same trial.

\section{Results}

\section{Latencies to look at the cue}

Trials in which infants did not look at the cue were not considered in the following analysis ( 68 trials out of 608 , i.e., $11.18 \%$ of the data). Out of the 540 remaining data points, five were identified as outliers (i.e., they were more than three standard deviations above the overall mean latency) and removed from the analysis.

In accordance with our prediction that snake pictures would improve attentional capture, a paired-sample $t$ test on the latencies to look at the cue revealed that infants oriented their gaze faster towards snake than flower cues, $t(18)=2.57, p=.019$, Cohen's $d=.59$ (for snake cues: $M=284 \mathrm{~ms}, S D=53$, for flower cues: $M=304 \mathrm{~ms}, S D=51$ ).

The average latency difference did not correlate with age, $r=.264, p>.10$.

\section{Latencies to look at the target}

Trials in which infants did not look at the cue (see prior analysis), did not look back at the subsequent central star, were not looking at the central star at the onset of the target, and trials in which eye movements were not made directly towards a target were not considered in the two following analyses (together, 128 trials out of 608 , i.e., $21.05 \%$ of the data). Out of the 474 remaining data points, seven were identified as outliers and removed from the analyses.

Table 1 displays the mean latencies of the first looks at the cued and the uncued targets as a function of the preceding cue.

A 2 (Cue: flower vs. snake) $\times 2$ (Gaze direction: cued vs. uncued target) repeated measures ANOVA was performed on the first look latencies toward the target. No effect or interaction were significant, all $p>.10$. Hence, contrary to our predictions, no cue facilitation effect was observed at the level of gaze latency.

\section{Proportion of orienting to the cued and uncued targets}

Table 2 displays the mean proportion of gaze orienting towards the cued and the uncued targets, as a function of the preceding cue.

A 2 (Cue) $\times 2$ (Gaze direction) repeated measures ANOVA was performed on the proportion of orienting toward the targets. This analysis revealed a main effect of Gaze direction, $F(1,18)=9.43$,
Table 2. Mean percentages of gaze orienting towards the cued and the uncued targets as a function of the type of cue presented before.

\begin{tabular}{|c|c|c|}
\hline & \multicolumn{2}{|c|}{ Direction of first look } \\
\hline & Cued target & Uncued target \\
\hline Flower cue & $63.7[57.6,69.8]$ & $36.3[30.2,42.5]$ \\
\hline Snake cue & $54.1[45.3,62.9]$ & $45.9[37.0,54.7]$ \\
\hline
\end{tabular}

Note. $n=19.95 \% \mathrm{Cls}$ are in brackets.

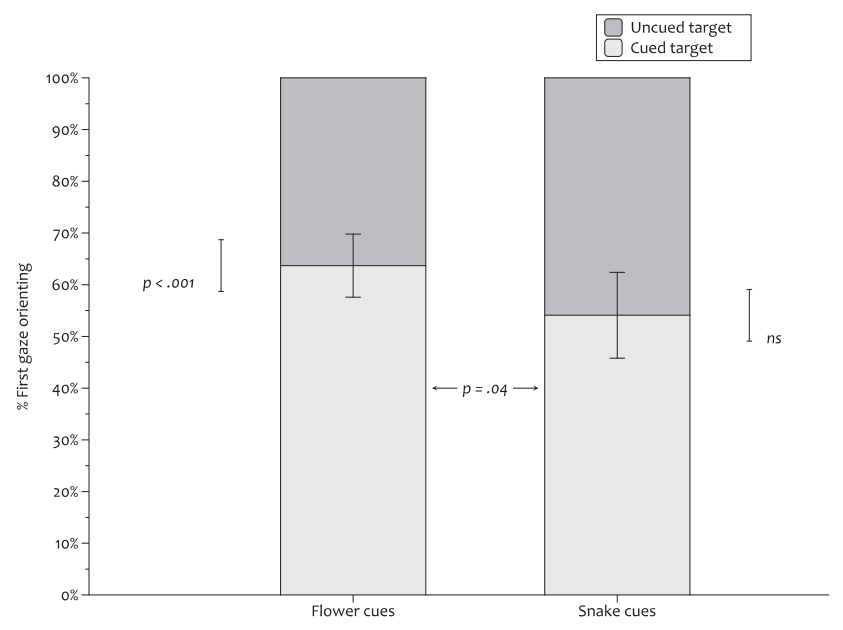

Note. $\mathrm{n}=19$

Figure 2. Proportion of orienting toward the cued target separately for threat-irrelevant (flower cues) and threat-relevant trials (snake cues).

$p=.007$, partial $\eta^{2}=.34$ : infants' first look was more often directed toward the cued than toward the uncued target (in $59 \%$ vs. $41 \%$ of the trials, $S D=12.7)$. In addition, the interaction between Gaze direction and Cue was also significant, $F(1,18)=$ $4.91, p=.04$, partial $\eta^{2}=.21$. Bonferroni-adjusted comparisons revealed that these proportions differed as a function of the preceding cue: When flower cues were presented, the proportion of first gaze oriented toward the cued target was significantly higher than the frequency of first gaze oriented toward the uncued target, $t(18)$ $=4.694, p<.001$; this was not the case when snake cues were presented, $t<1$. Moreover, although infants' first looks were directed toward the cued target in $63.7 \%$ of the trials in which a flower cue was presented $(S D=12.6)$, this proportion dropped to $54.1 \%$ of the trials in which a snake cue was presented $(S D=18.3)$, $p=.04$ (see Figure 2).

The mean difference between the proportion of first looks directed to the cued target when flower and snake cues were presented did not correlate with age, $r=.222, p>.10$.

In accordance with our predictions, the presentation of a snake modulated the cue facilitation effects observed when flowers were presented.

\section{Discussion}

Using a variant of the Posner's cuing paradigm (Posner, 1980), we investigated the existence of a threat-dependent functional link between the "what" and the "where" visual subsystems in infancy. Specifically, we examined whether and how spatial orienting is influenced by the detection of snakes in the visual environment. 
We observed that, when presented with peripheral cues, 7-11month-old infants turned more quickly towards snakes than flowers. These findings add to the body of evidence showing attentional capture by pictures of snakes in infancy (DeLoache \& LoBue, 2009; LoBue \& DeLoache, 2010). They also extend these results by demonstrating that this grabbing of attention occurs under different presentation conditions. Specifically, although previous studies used pairs of pictures made up of one threat-relevant and one threat-irrelevant pictures (i.e., competing for attentional resources), pictures were presented in isolation in the current study and elicited exogenous overt attentional shifts towards their peripheral location, be they flowers or snakes. The present results thus show that the detection of a snake in the visual periphery would speed up the automatic engagement of attention towards its location.

In addition to these effects on attentional capture, central to the current study is the finding that the presentation of peripheral snake cues modulated spatial orienting towards the subsequent bilateral targets. While infants oriented their first gaze preferentially towards the cued than the uncued target after flower cues (i.e., facilitation effects ${ }^{4}$ ), when cues were pictures of snakes, infants oriented as much towards the cued than the uncued target (i.e., no facilitation effect). Detecting a snake (an ability linked to the "what" subsystem) would thus influence attentional orienting in the visual space (relying on the "where" subsystem) in such a way that subsequent stimuli would be differentially attended to depending on their location.

Although at first sight it might seem counterintuitive that infants orient less towards the cued location after having detected a threatrelevant stimulus rather than a neutral one, this can be explained in two ways. First, these data could reflect an avoidance reaction when detecting a snake, so that attention would be automatically diverted from that location. However, in that case one would have expected that infants orient their attention preferentially towards the opposite side of space. Rather, we observed that, when a snake was detected, infants oriented their attention as much towards the cued than towards the uncued location. This pattern of results is more compatible with an interpretation in terms of a broadening of attention. Detecting the snake in the grass would force us to enlarge our attentional focus in order to consider ways to escape from the threat and check for the presence of other potential dangers in the vicinity of the detected snake. This would lead to attention being directed as much to the threat location as to other locations.

It is worth noting that these facilitation effects were observed when considering the orienting behaviour as a dependent variable, not when examining gaze latencies (though these data were in the same direction). The absence of cuing effects on gaze latencies is not an isolated phenomenon in infant studies (Harman et al., 1994). In fact, these would not be an optimal dependent measure for detecting differences between cued and uncued targets since infants usually show slow and variable responses (Varga, Frick, Kapa, \& Dengler, 2010), even more so when SOAs are long. It may not be surprising then that the threat-dependent functional link between the "what" and "where" infants' visual subsystems was not observed at the level of gaze orienting latency. Further studies should nevertheless confirm the lack of cuing effect on saccade latencies in a larger group of infants.

Hence, together with studies in lab-reared monkeys (Shibasaki \& Kawai, 2009), the current and previous infant studies convincingly demonstrate that no prior experience with snakes or knowledge about the danger they may represent are required to show attentional biases linked to pictures of snakes. Our results are therefore in line with the claim that primates would have an evolved bias for the rapid detection of threat-relevant stimuli, and snakes in particular. They are consistent with the Snake Detection Theory positing that snake detection by the human visual system improved through natural selection as these animals have been preying on mammals for tens of millions of years (Isbell, 2006, 2009). They are also in line with the proposal of an evolved, relatively encapsulated fear module in the brain that would be selectively sensitive to and automatically activated by evolutionary threat-relevant stimuli, allowing their rapid detection (Öhman \& Mineka, 2001, 2003). Although it is tempting to consider such biases as innate given that they are present early in life, similar studies should be run in newborns (who truly have no experience of their visual world) before any conclusion can be drawn.

What are the mechanisms underlying these biases in infancy? While the threat value assigned to fear-relevant stimuli would be a critical factor to activate the fear module in adults (Öhman et al., 2001), 7-11-month-old infants have likely not yet labelled snakes as being threatening. This assumption is supported by the fact that infants do not show any evidence of actual fear when exposed to videos of moving snakes (DeLoache \& LoBue, 2009; Thrasher \& LoBue, 2016). Most probably, in the current study, infants' attention was rather captured by low-level visual features characterizing snakes (LoBue, Rakison, \& DeLoache, 2010; Rakison \& Derringer, 2008). Natural selection would indeed have fostered the evolution of the primates' visual system such that it rapidly detects perceptual features that are associated with phylogenetically threat-relevant stimuli (Cave \& Batty, 2006; Isbell, 2006, 2009). Accordingly, in Öhman's model of fear activation, external stimuli would first pass through a "features detector" module, which would automatically and unconsciously detect threat based on simple perceptual features (Öhman, 1993). For snakes in particular, such a perceptual feature would be their curvilinear, often coiled shape (LoBue, 2014; LoBue $\&$ DeLoache, 2011). Snakes' curvy shape would indeed determine snake detection in children and adults, while their bright coloration, specific natural backgrounds and facial traits would have no impact (LoBue \& DeLoache, 2011), at least under the conditions and in the age ranges tested. What features drive this perceptual bias in the first years of life remains unstudied. Still, it is possible that the underlying mechanisms and specific features follow a developmental trajectory. For instance, although the coiled shape would be sufficient to elicit an attentional bias in toddlers and adults, infants could be sensitive to a combination of features such as curvy shape, bright colours and contrasts (three attributes of our set of stimuli). Moreover, cognitive factors could play an increasing role in the occurrence of the effects of snake features presentation across development (for evidence in adults, see LoBue, 2014). Putting aside the threat value children will learn to assign to snakes at one point in their development (probably after having entered school), the mere familiarity with the stimuli, both threatrelevant and threat-irrelevant, presented in these studies (acquired through picture books for instance) could determine the occurrence of the bias. Further studies should carefully control these parameters in order to determine which specific features infants are sensitive to, and how their day-to-day experience might influence the occurrence of the bias.

In conclusion, the present study provides compelling evidence that human infants are biased for the fast detection of snakes, and that the connection between the "what" and "where" visual subsystems is efficient in 7-11-month-old infants for the processing of 
threat-relevant stimuli. Indeed, detecting snakes in their visual environment modulates infants' gaze behaviour, most probably by enlarging their attentional focus. Further studies should confirm the perceptual nature of this bias and aim at identifying the specific features that are crucial for the detection of snakes and other evolutionary threat-relevant stimuli.

\section{Acknowledgments}

We thank Vanessa LoBue for providing us with the pictures of flowers and snakes that were used in this experiment. We thank Emeline Boursain and Julia Spreer for having coded the videos of the present study and its preliminary version.

\section{Funding}

The authors disclosed receipt of the following financial support for the research, authorship, and/or publication of this article: This work was supported by a FRS - FNRS grant (F.4524.10). The first author was a Post-Doctoral Researcher of the Fonds de la Recherche Scientifique-FNRS.

\section{Notes}

1. We considered this age range reasonable since previous studies reported no age effect on the detection of threat-relevant stimuli in this age group (DeLoache \& LoBue, 2009; LoBue \& DeLoache, 2010; LoBue, Buss, Taber-Thomas, \& PérezEdgar, 2016).

2. Pilot testing showed that these stimuli durations were the most suitable in order to foster overt attentional shifts to the cue and to ensure that attention was back to the centre of the screen when the bilateral target was presented.

3. Latencies to look at the cue and at the target were calculated from the onset of the stimulus to the infant's first look (i.e., first saccade) toward it rather than to the infant's first fixation on it. This was indeed a purer measure of infant's attentional orienting from the centre of the screen to the peripheral stimulus given (1) the use of video coding that prevents any precise information about where (and when) exactly the baby is looking at, and given (2) the fleetingness of the cue that had most of the time disappeared when the baby's gaze reached its location.

4. One might wonder why we did not observe inhibition instead of facilitation effects when emotionally neutral, threat-irrelevant cues were presented. Indeed, the use of long SOAs and a central stimulus between the cue and the target in order to disengage attention from the location of the cue typically favour the occurrence of the Inhibition of Return phenomenon (IOR; Posner \& Cohen, 1984), namely the propensity to preferentially orient towards uncued than cued (i.e., recently attended) targets. However, given the eccentricity of our cues $\left(30^{\circ}\right)$, infants' eye movements were clearly multisaccadic, and it has been argued that IOR occurs following cues to which single and accurate saccades have been made (Butcher, Kalverboer, \& Geuze, 1999; Rafal, Calabresi, Brennan, \& Sciolto, 1989). Moreover, although long SOAs would favour inhibition over facilitation effects, no IOR would be observed in infants for inter-trial intervals (ITIs) beyond 3.5 seconds (Clohessy, Posner, Rothbart, \& Vecera, 1991). Clearly, ITIs exceeded this limit in our experimental design. Hence, range of eccentricity and long ITIs could be responsible (at least partially) for the absence of IOR in the present study.

\section{References}

Bertels, J., Kolinsky, R., Bernaerts, A., \& Morais, J. (2011). Effects of emotional spoken words on exogenous attentional orienting. Journal of Cognitive Psychology, 23(4), 435-452.

Bertels, J., Kolinsky, R., \& Morais, J. (2010). Emotional valence of spoken words influences the spatial orienting of attention. Acta Psychologica, 134(3), 264-278.

Brainard, D. H. (1997). The psychophysics toolbox. Spatial Vision, 10, 433-436.

Bushneil, I., Sai, F., \& Mullin, J. (1989). Neonatal recognition of the mother's face. British Journal of Developmental Psychology, 7(1), 3-15.

Butcher, P. R., Kalverboer, A. F., \& Geuze, R. H. (1999). Inhibition of return in very young infants: A longitudinal study. Infant Behavior and Development, 22, 303-319.

Cave, K. R., \& Batty, M. J. (2006). From searching for features to searching for threat: Drawing the boundary between preattentive and attentive vision. Visual Cognition, 14(4-8), 629-646.

Clohessy, A. B., Posner, M. I., Rothbart, M. K., \& Vecera, S. P. (1991). The development of inhibition of return in early infancy. Journal of Cognitive Neuroscience, 3(4), 345-350.

DeLoache, J. S., \& LoBue, V. (2009). The narrow fellow in the grass: Human infants associate snakes and fear. Developmental Science, 12(1), 201-207.

Erlich, N., Lipp, O. V., \& Slaughter, V. (2013). Of hissing snakes and angry voices: Human infants are differentially responsive to evolutionary fear-relevant sounds. Developmental Science, 16(6), 894-904.

Harman, C., Posner, M. I., Rothbart, M. K., \& Thomas-Thrapp, L. (1994). Development of orienting to locations and objects in human infants. Canadian Journal of Experimental Psychology, 48(2), 301-318.

Hayakawa, S., Kawai, N., \& Masataka, N. (2011). The influence of color on snake detection in visual search in human children. Scientific Report, 1, 80.

Isbell, L. A. (2006). Snakes as agents of evolutionary change in primate brains. Journal of Human Evolution, 51(1), 1-35.

Isbell, L. A. (2009). The fruit, the tree, and the serpent: Why we see so well. Cambridge, MA: Harvard University Press.

Johnson, M. (1994). Visual attention and the control of eye movements in early infancy. In C. Umiltà \& M. Moscovitch (Eds.), Attention and performance $X V$ : Conscious and nonconscious information processing (pp. 291-310), Cambridge, MA: The MIT Press.

Johnson, M. H., Mareschal, D., \& Csibra, G. (2008). The development and integration of the dorsal and ventral visual pathways in object processing. In C. A. Nelson \& M. Luciana (Eds.), Handbook of developmental cognitive neuroscience (2nd ed., pp. 467-478). Cambridge, MA: The MIT Press.

Johnson, M. H., Dziurawiec, S., Ellis, H., \& Morton, J. (1991). Newborns' preferential tracking of face-like stimuli and its subsequent decline. Cognition, 40(1), 1-19.

Johnson, M. H., \& Tucker, L. A. (1996). The development and temporal dynamics of spatial orienting in infants. Journal of Experimental Child Psychology, 63(1), 171-188.

Lipp, O. V., \& Derakshan, N. (2005). Attentional bias to pictures of fear-relevant animals in a dot probe task. Emotion, 5(3), 365-369.

Lipp, O. V., Derakshan, N., Waters, A. M., \& Logies, S. (2004). Snakes and cats in the flower bed: Fast detection is not specific to pictures of fear-relevant animals. Emotion, 4, 233-250. 
LoBue, V. (2014). Deconstructing the snake: The relative roles of perception, cognition, and emotion on threat detection. Emotion, 14(4), 701-711.

LoBue, V., Buss, K. A., Taber-Thomas, B. C., \& Pérez-Edgar, K. (2016). Developmental differences in infants' attention to social and nonsocial threats. Infancy. Advance online publication. doi: 10.1111/infa. 12167

LoBue, V., \& DeLoache, J. S. (2008). Detecting the snake in the grass: Attention to fear-relevant stimuli by adults and young children. Psychological Science, 19(3), 284-289.

LoBue, V., \& DeLoache, J. S. (2010). Superior detection of threat-relevant stimuli in infancy. Developmental Science, 13(1), 221-228.

LoBue, V., \& DeLoache, J. S. (2011). What's so special about slithering serpents? Children and adults rapidly detect snakes based on their simple features. Visual Cognition, 19(1), 129-143.

LoBue, V., \& Matthews, K. (2014). The snake in the grass revisited: An experimental comparison of threat detection paradigms. Cognition and Emotion, 28, 22-35.

LoBue, V., Matthews, K., Harvey, T., \& Stark, S. L. (2014). What accounts for the rapid detection of threat? Evidence for an advantage in perceptual and behavioral responding from eye movements. Emotion, 14, 816-823.

LoBue, V., Rakison, D. H., \& DeLoache, J. S. (2010). Threat perception across the life span evidence for multiple converging pathways. Current Directions in Psychological Science, 19(6), 375-379.

Masataka, N., Hayakawa, S., \& Kawai, N. (2010). Human young children as well as adults demonstrate 'superior' rapid snake detection when typical striking posture is displayed by the snake. PLoS One, 5(11), e15122.

Milner, A. D., \& Goodale, M. A. (1998). The visual brain in action. Oxford: Oxford University Press.

Öhman, A. (1993). Fear and anxiety as emotional phenomena: Clinical phenomenology, evolutionary perspectives, and informationprocessing mechanisms. In M. H. Lewis \& J. M. Haviland (Eds.), Handbook of emotions (pp. 511-536). New York, NY: The Guilford Press.

Öhman, A., Flykt, A., \& Esteves, F. (2001). Emotion drives attention: Detecting the snake in the grass. Journal of Experimental Psychology: General, 130(3), 466-478.

Öhman, A., \& Mineka, S. (2001). Fears, phobias, and preparedness: Toward an evolved module of fear and fear learning. Psychological Review, 108(3), 483-522.

Öhman, A., \& Mineka, S. (2003). The Malicious Serpent: Snakes as a prototypical stimulus for an evolved module of fear. Current Directions in Psychological Science, 12(1), 5-9.

Peltola, M. J., Leppanen, J. M., Palokangas, T., \& Hietanen, J. K. (2008). Fearful faces modulate looking duration and attention disengagement in 7-month-old infants. Developmental Science, $11(1), 60-68$.

Penkunas, M. J., \& Coss, R. G. (2013a). A comparison of rural and urban Indian children's visual detection of threatening and nonthreatening animals. Developmental Science, 16, 463-475.

Penkunas, M. J., \& Coss, R. G. (2013b). Rapid detection of visually provocative animals by preschool children and adults. Journal of Experimental Child Psychology, 114(4), 522-536.

Posner, M. I. (1980). Orienting of attention. Quarterly Journal of Experimental Psychology, 32(1), 3-25.

Posner, M. I., \& Cohen, Y. (1984). Components of visual orienting. Attention and performance $X$ : Control of language processes, 32, 531-556.

Rafal, R. D., Calabresi, P. A., Brennan, C. W., \& Sciolto, T. K. (1989). Saccade preparation inhibits reorienting to recently attended locations. Journal of Experimental Psychology: Human Perception and Performance, 15, 673-685.

Rakison, D. H., \& Derringer, J. (2008). Do infants possess an evolved spider-detection mechanism? Cognition, 107(1), 381-393.

Reid, V. M., Striano, T., Kaufman, J., \& Johnson, M. H. (2004). Eye gaze cueing facilitates neural processing of objects in 4-month-old infants. Neuroreport, 15(16), 2553-2555.

Shibasaki, M., \& Kawai, N. (2009). Rapid detection of snakes by Japanese monkeys (Macaca fuscata): An evolutionarily predisposed visual system. Journal of Comparative Psychology, 123(2), 131-135.

Soares, S. C., Lindström, B., Esteves, F., \& Öhman, A. (2014). The hidden snake in the grass: Superior detection of snakes in challenging attentional conditions. PLoS one, 9, el14724.

Stiles, J., Paul, B., \& Ark, W. (2008). The development of visuospatial processing. In C. A. Nelson \& M. Luciana (Eds.), Handbook of developmental cognitive neuroscience (2nd ed., pp. 521-540). Cambridge, MA: The MIT Press.

Stormark, K. M., Nordby, H., \& Hugdahl, K. (1995). Attentional shifts to emotionally charged cues: Behavioural and ERP data. Cognition \& Emotion, 9(5), 507-523.

Thrasher, C., \& LoBue, V. (2016). Do infants find snakes aversive? Infants' physiological responses to "fear-relevant" stimuli. Journal of Experimental Child Psychology, 142, 382-390.

Valenza, E., Simion, F., \& Umiltà, C. (1994). Inhibition of return in newborn infants. Infant Behavior and Development, 17(3), 293-302.

Van Le, Q., Isbell, L. A., Matsumoto, J., Nguyen, M., Hori, E., Maior, R. S., . . Nishijo, H. (2013). Pulvinar neurons reveal neurobiological evidence of past selection for detection of snakes. Proceedings of the National Academy of Sciences of the United States of America, 110(47), 19000-19005.

Varga, K., Frick, J. E., Kapa, L. L., \& Dengler, M. J. (2010). Developmental changes in inhibition of return from 3 to 6 months of age. Infant Behavior and Development, 33(2), 245-249. 